\title{
High-Dose-Rate Electron-Beam Dosimetry Using an Advanced Markus Chamber with Improved lon- Recombination Corrections
}

\author{
Dong Hyeok Jeong ${ }^{\mathbb{1}}$, Manwoo Lee ${ }^{(\mathbb{D}}$, Heuijin Lim ${ }^{(\mathbb{D}}$, Sang Koo Kang ${ }^{\mathbb{1}}$, Kyoung Won Jang ${ }^{(\mathbb{D}}$ \\ Research center, Dongnam Institute of Radiological and Medical Sciences, Busan, Korea
}

Received 19 August 2020

Revised 19 October 2020

Accepted 23 October 2020

\section{Corresponding author}

Kyoung Won Jang

(kko988@hotmail.com)

Tel: 82-51-720-5813

Fax: 82-51-720-5826
Purpose: In ionization-chamber dosimetry for high-dose-rate electron beams-above 20 $\mathrm{mGy} /$ pulse -the ion-recombination correction methods recommended by the International Atomic Energy Agency (IAEA) and the American Association of Physicists in Medicine (AAPM) are not appropriate, because they overestimate the correction factor. In this study, we suggest a practical ion-recombination correction method, based on Boag's improved model, and apply it to reference dosimetry for electron beams of about $100 \mathrm{mGy} /$ pulse generated from an electron linear accelerator (LINAC).

Methods: This study employed a theoretical model of the ion-collection efficiency developed by Boag and physical parameters used by Laitano et al. We recalculated the ion-recombination correction factors using two-voltage analysis and obtained an empirical fitting formula to represent the results. Next, we compared the calculated correction factors with published results for the same calculation conditions. Additionally, we performed dosimetry for electron beams from a 6 $\mathrm{MeV}$ electron LINAC using an Advanced Markus ${ }^{\boxplus}$ ionization chamber to determine the reference dose in water at the source-to-surface distance $(S S D)=100 \mathrm{~cm}$, using the correction factors obtained in this study.

Results: The values of the correction factors obtained in this work are in good agreement with the published data. The measured dose-per-pulse for electron beams at the depth of maximum dose for SSD=100 $\mathrm{cm}$ was $115 \mathrm{mGy} /$ pulse, with a standard uncertainty of $2.4 \%$. In contrast, the $k_{\mathrm{s}}$ values determined using the IAEA and AAPM methods are, respectively, $8.9 \%$ and $8.2 \%$ higher than our results.

Conclusions: The new method based on Boag's improved model provides a practical method of determining the ion-recombination correction factors for high dose-per-pulse radiation beams up to about $120 \mathrm{mG} /$ pulse. This method can be applied to electron beams with even higher doseper-pulse, subject to independent verification.

Keywords: Ion recombination correction factor, Dose-per-pulse, Advanced Markus chamber, Boag model, Electron beam dosimetry

\section{Introduction}

Typically, electron-beam radiotherapy currently utilizes two dose-rate ranges: around $1 \mathrm{mGy} /$ pulse $(\sim 6 \mathrm{~Gy} / \mathrm{min})$ for conventional radiotherapy and up to about $100 \mathrm{mGy} /$ pulse ( $40 \mathrm{~Gy} / \mathrm{min})$ for intraoperative radiotherapy [1]. Recently, ultra-high dose rates-exceeding $200 \mathrm{mGy} /$ pulse (>40 Gy/s) have been used in pre-clinical studies for FLASH radio-

Copyright (c) 2020 Korean Society of Medical Physics

@This is an Open-Access article distributed under the terms of the Creative Commons Attribution Non-Commercial License (http://creativecommons.org/licenses/by$\mathrm{nc} / 4.0$ ) which permits unrestricted non-commercial use, distribution, and reproduction in any medium, provided the original work is properly cited. 
therapy $[2,3]$. At these increased dose rates-especially at dose rates over $10 \mathrm{mGy} / \mathrm{min}$-ion-recombination is a very significant process in dosimetry that uses an ionization chamber [4]. However, current methods for correcting for ion recombination-known as the "two-voltage technique," which involves protocols recommended by the International Atomic Energy Agency (IAEA) or the American Association of Physicists in Medicine (AAPM) - only applies to dose rates below about $20 \mathrm{mGy} /$ pulse. As pointed out in several previous works, these conventional methods overestimate the ion-recombination correction factor for high dose-per-pulse (DPP) radiation beams [5].

Boag studied the ion-recombination process theoretically since the 1950s, and he contributed to the development of the current correction methods [6]. In 1996, he published three improved models to correct for ion recombination in high-dose-rate pulsed radiation beams [6]. Currently, Boag's models are the recognized reference standard for high-DPP radiation dosimetry. Laitano et al. [7] subsequently measured electron beams of 20-120 mGy/pulse and solved the relevant equations for Boag's improved models. They used an iterative numerical method to determine the ion-recombination correction factors from the two measured charges $\left(M_{1}, M_{2}\right)$ obtained at two polarization voltages $\left(V_{1}, V_{2}\right)$ [7].

Boag's model provides information about the ion-collection efficiency and gives a method of calculating the recombination correction factors for high-DPP beams. However, applying for practical dosimetry is inconvenient due to the difficulty of the numerical analysis. For high-DPP beams, the relationship between the DPP and the ion-recombination correction factors has been investigated using absolute dosimetry or radiochromic films, but it has not yet been applied in practice $[5,8]$.

Through Boag's improved model and the work of Laitano et al. [7] cited above, ion-recombination correction factors can be determined directly in terms of the one variable, $M_{1} /$ $M_{2}$, as in the conventional two-voltage technique, but without requiring iterative methods. In the present study, by reconstructing these calculated results, we developed a fitting formula that yields the ion-recombination correction factor using only two measured values. This fitting formula exhibits a similar shape to those provided by the TRS-398 or TG51 protocols, and it is useful for practical applications. We verified the results by recalculating the ion-recombination correction factors published by Laitano et al. [7]. Then, we applied this method to determine the reference dose rate for electron beams generated by an electron linear accelerator (LINAC). We used the Advanced Markus ${ }^{\circledR}$ plane-parallel ionization chamber (PTW, Freiburg, Germany) with $1 \mathrm{~mm}$ electrode spacing throughout this study [9].

\section{Materials and Methods}

\section{Ion-recombination correction}

The ion-recombination correction factor is used to correct the response of an ionization chamber for the lack of complete charge collection, which is due to the recombination of ions exhibiting opposite charges during transit to each electrode. For positive ions, the ion-collection efficiency $f$ is given by the ratio of the collected charge $M$ to the total charge produced, $M_{0}: f=M / M_{0}$. Then, the ionrecombination correction factor is defined as $k_{\mathrm{s}}=1 / f[6,10]$.

Because interactions between the ions themselves-or between ions and neutral molecules-in an electric field are complicated, determining $f$ or $k_{\mathrm{s}}$ exactly is difficult, so $k_{\mathrm{s}}$ is currently obtained from empirical formulas [10]. In the IAEA code of practice (the TRS-398 protocol) for pulsed radiation beams, the ion-recombination correction factor $k_{s}$ is given by [11]

$$
k_{s}=a_{0}+a_{1}\left(\frac{M_{1}}{M_{2}}\right)+a_{2}\left(\frac{M_{1}}{M_{2}}\right)^{2},
$$

where $a_{0}, a_{1}$, and $a_{2}$ are constants that depend on the ratio $V_{1} / V_{2}$ of the two polarizing voltages. For $V_{1} / V_{2}=2$, the values of the constants are $a_{0}=2.337, a_{1}=-3.636$, and $a_{2}=2.229$. The quantity $M_{1} / M_{2}$ is the ratio of the charges measured at the two polarizing voltages $V_{1}$ and $V_{2}$. Similarly, in the AAPM TG-51 protocol, the ion-recombination correction factor $P_{i o n}\left(=k_{\mathrm{s}}\right)$ is given by [12]

$$
P_{\text {ion }}=\left(\frac{1-V_{1} / V_{2}}{M_{1} / M_{2}-V_{1} / V_{2}}\right) \text {. }
$$

Both of these methods are based on Boag's early model (1950), which assumes a linear dependence of $1 / M$ on $1 / V$ [11]. According to that model, the correction factor is deter- 
mined by extrapolating the $1 / M$ vs. $1 / V$ plot to determine the value $1 / M_{0}$ obtained when $1 / V=0$, where $M_{0}$ is the saturated charge for which $f=1$. By estimating $M_{0}$, one can thus obtain the ion-correction efficiency $f=M_{1} / M_{0}$ or the ionrecombination correction factor $k_{\mathrm{s}}=M_{0} / M_{1}$ [13].

The quantity $f$ declines from 1 to 0 with increasing DPP, and for clinical electron beams (DPP $<1 \mathrm{mGy}$ ), the recombination correction factor can be determined using the current methods that apply the dosimetry protocols of the IAEA and AAPM. However, because free electrons that do not contribute to the production of negative ions increase with increasing DPP, eventually the value of $f$ does not continue to decrease significantly. Thus, the current methods are not appropriate for cases with high DPP, especially DPP>20 mGy $[6,7]$.

Therefore, in order to incorporate free electrons into the determination of the ion-collection efficiency, Boag proposed three improved models, where the third model (denoted by $f^{\prime \prime \prime}$ ) was introduced as a practical correction method for high-DPP electron beams [6]. The ion collection efficiency ( $\left.f^{\prime \prime}\right)$ in Boag's third model is given by [6]

$$
f^{\prime \prime}=\lambda+\frac{1}{u} \ln \left[1+\frac{e^{\lambda(1-\lambda) u}-1}{\lambda}\right]
$$

where $\lambda=1-\sqrt{1-p}, p$ is the free-electron fraction of the total electrons produced by a beam pulse, the dimensionless parameter $u=\mu r d^{2} / V$, where $\mu$ depends on the ionic recombination coefficient and the ion mobility, $r$ is the charge density of positive ions initially generated by the beam pulse, $d$ is the electrode spacing, and $V$ is the polarizing voltage $[6,7]$.

Equation (3) cannot be solved directly due to the difficulty in determining the parameter $u$. However, if $f_{1}$ and $f_{2}$ are the ion-collection efficiencies for the charges $M_{1}$ and $M_{2}$ measured at the two polarizing voltages $V_{1}$ and $V_{2}$, respectively, then the ratio $f_{1} / f_{2}=M_{1} / M_{2}$ can be expressed a function of $u_{1}$ by using $u_{1} / u_{2}=V_{2} / V_{1}$ :

$$
\frac{f_{1}\left(u_{1}\right)}{f_{2}\left(u_{1}\right)}=\frac{\lambda_{1}+\frac{1}{u_{1}} \ln \left[1+\frac{e^{\lambda_{1}\left(1-\lambda_{1}\right) u_{1}}-1}{\lambda_{1}}\right]}{\lambda_{2}+\frac{1}{\left(\frac{V_{1}}{V_{2}}\right) u_{1}} \ln \left[1+\frac{e^{\lambda_{2}\left(1-\lambda_{2}\right)\left(\frac{V_{1}}{V_{2}}\right) u_{1}}-1}{\lambda_{2}}\right]}=\frac{M_{1}}{M_{2}} .
$$

Then, one can determine $u_{1}$ iteratively using a computer program and the measured value of $M_{1} / M_{2}$. The correction factor $k_{\mathrm{s}}=1 / f 1$ can be determined by substituting the resulting value of $u_{1}$ into the expression for $f_{1}$. This is the method proposed by Boag et al. (1996) [6] and implemented by Laitano et al. (2006) [7]. However, although this method can be used to determine $k \mathrm{~s}$, applying in practice is inconvenient.

Conversely, because the single variable $u_{1}$ determines both $M_{1} / M_{2}$ and $f_{1}$ simultaneously, the correction factor $k_{\mathrm{s}}=1 / f_{1}$ can be determined as a function of $M_{1} / M_{2}$ without iteration by utilizing pre-calculated values of $M_{1} / M_{2}$ and $f_{1}$ as functions of $u_{1}$. Thus, the quantity $f_{1}$ can be obtained as a function of $M_{1} / M_{2}$ for practical use by fitting. Our study used this new method with the new equations based on the Boag model, to measure high-DPP electron beams produced by an electron LINAC.

\section{Verification of the calculations}

Laitano et al. [7] investigated Boag's three improved models and published the ion-recombination factors determined for six types of commercial plane-parallel ionization chambers for various polarization voltages. For comparison with our method, we selected 22 of the data points obtained by Laitano et al. [7] for comparison. The selected data cover the range $0.1-70 \mathrm{mGy} /$ pulse and include values of $M_{1} / M_{2}$ up to about 2, as measured at 10 voltage ratios with an Exradin A12 ionization chamber. We solved equation (4) for the given $M_{1} / M_{2}$ values and two polarization voltages, and we fitted the $k_{\mathrm{s}}$ values as a function of $M_{1} / M_{2}$ with second-order polynomial functions. We employed the same physical parameters used by Laitano et al. [7] in our calculations of equation (4).

\section{Electron-beam measurements}

To determine the reference dose in water at source-tosurface distance $(\mathrm{SSD})=100 \mathrm{~cm}$, we performed dosimetry for electron beams from a $6 \mathrm{MeV}$ electron LINAC using an Advanced Markus ${ }^{\circledR}$ ionization chamber, as shown in Fig 1. The electron LINAC used in this study is the prototype developed by the Dongnam Institute of Radiological and Medical Science in collaboration with the Pohang Accelera- 

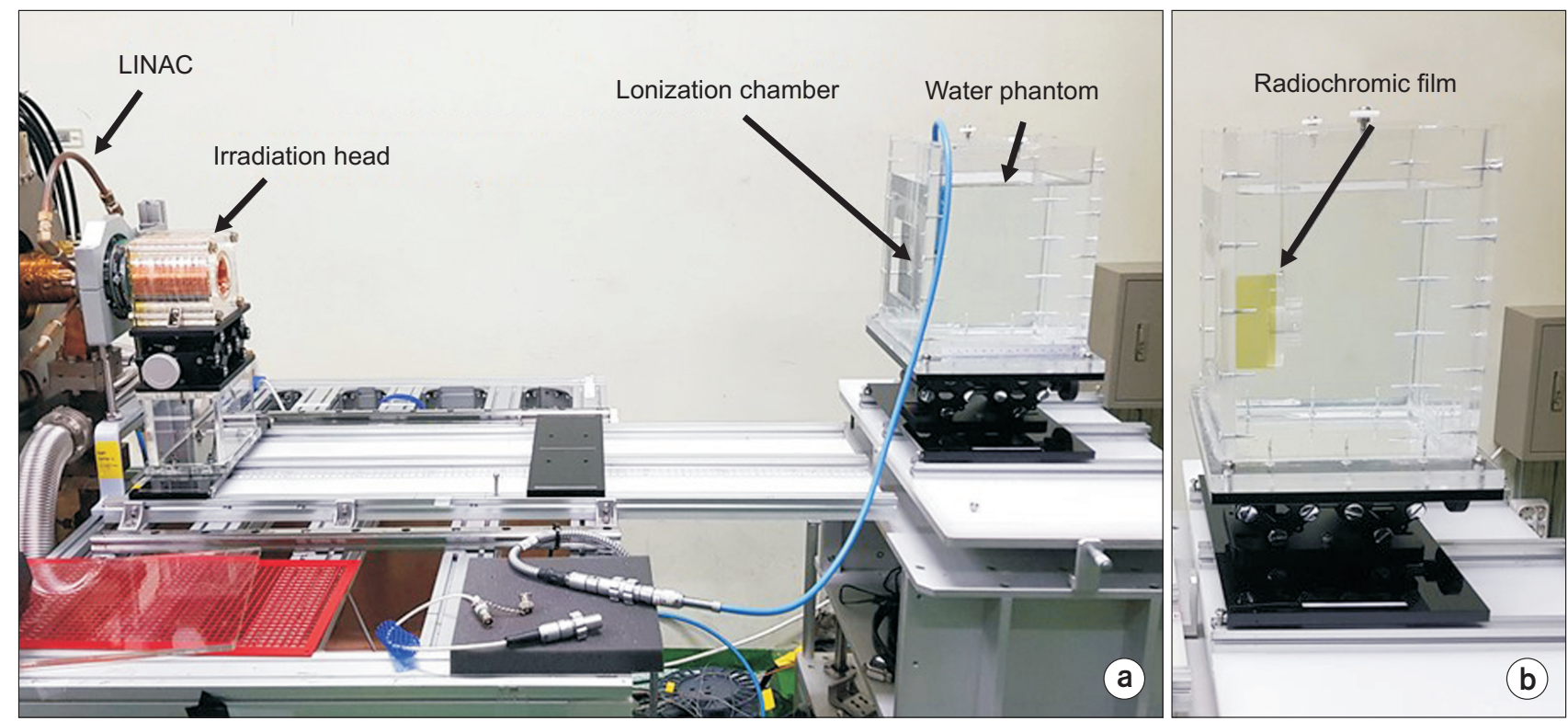

Fig. 1. Experimental setup (a) for reference dosimetry using an Advanced Markus ${ }^{\circledR}$ chamber and (b) for percentage depth dose measurements using a radiochromic film in water. LINAC, linear accelerator.

tor Laboratory [14]. Because the electron energy depends on the heating current of the electron gun, this experiment was performed at about $6 \mathrm{MeV}$ by adjusting the heating current [15].

An electron-irradiation device was used for the electronbeam irradiations, as shown in Fig. 1. It consists of specially designed scattering foils and collimators to generate an optimal electron beam for FLASH preclinical studies. We do not discuss its detailed geometry in the present work because the irradiation device is still under study.

The ionization chamber was calibrated in terms of water equivalents using a Co-60 reference beam. We applied the TRS-398 protocol to determine the dose in water [11]. The reference point of the ionization chamber was positioned at $z_{\text {ref }}=0.6 R_{50}-0.1 \mathrm{~cm}$, where $R_{50}$ is the half-value depth in water [11]. In this work, we determined $R_{50}$ by measuring the percentage depth dose (PDD) curve using a radiochromic film. The ionization measurements were performed at polarization voltages of $400 \mathrm{~V}$ and $200 \mathrm{~V}$ for 100 electron-beam pulses at the repetition rate of $50 \mathrm{~Hz}$ using pulse-mode control of the pulse-modulator system [16]. We applied the values of $k_{\mathrm{s}}$ calculated with the new method to correct the measured charge, and we also applied environmental and polarity corrections according to the TRS-398 protocol [11].

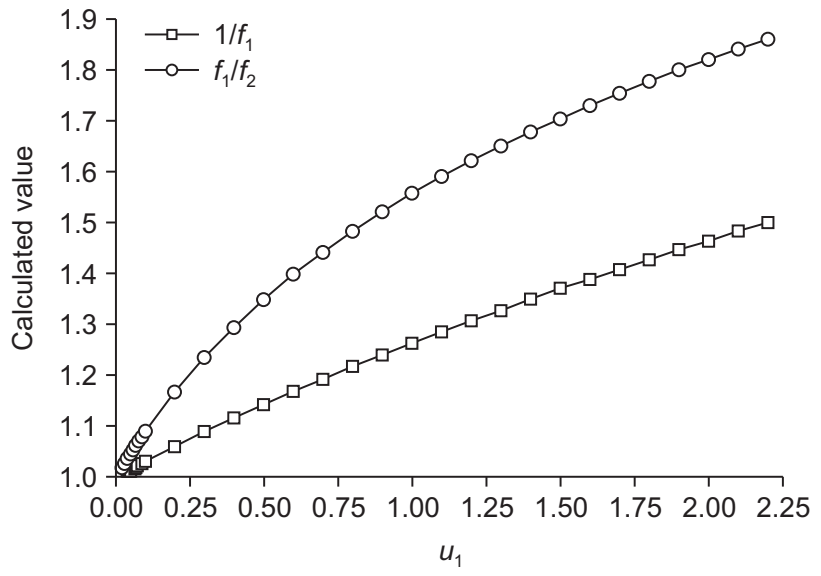

Fig. 2. Ratio of the ion-collection efficiency $\left(f_{1} / f_{2}\right)$ and the correction factor $\left(1 / f_{1}\right)$ calculated as a function of $u_{1}$ using Boag's model.

\section{Results}

\section{Calculation and verification}

Fig. 2 shows $f_{1} / f_{2}$ and $1 / f_{1}$ calculated as a function of $u_{1}$ for $V_{1} / V_{2}=400 / 200$ using equations (3) and (4) for the Advanced Markus $^{\circledR}$ chamber, a plane-parallel ionization chamber with an electrode spacing of $1 \mathrm{~mm}$. The value of $k_{\mathrm{s}}$ can be determined as a function of $M_{1} / M_{2}$, because $k_{\mathrm{s}}=1 / f_{1}$ and $M_{1} /$ $M_{2}=f_{1} / f_{2}$.

In this manner, we calculated $k_{\mathrm{s}}$ for the 22 selected data 

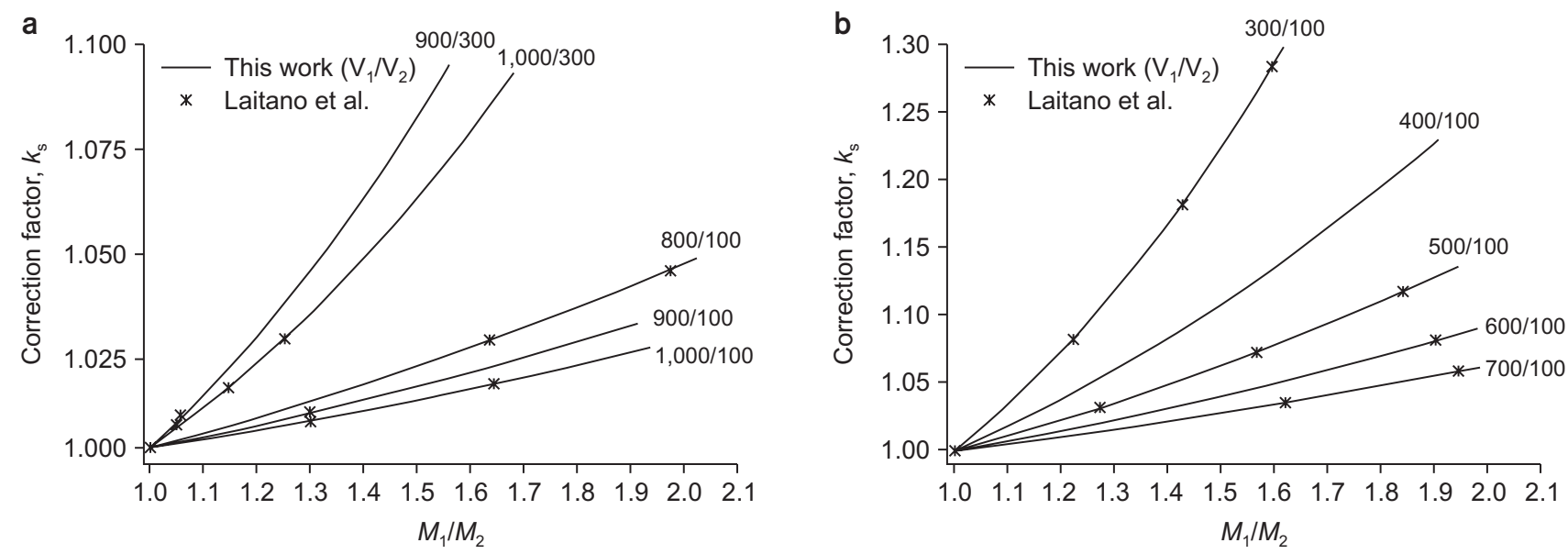

Fig. 3. Comparison of the ion-recombination correction factors recalculated in this work with data published by Laitano et al. [7] for 5 polarizing voltage ratios. See text for the explanation of the difference between graphs (a) and (b).

points, and the calculated results are shown in Fig. 3 together with the results of Laitano et al. [7]. Here, Fig. 3a and Fig. 3b, respectively, are for cases with small and with large slopes of $k_{\mathrm{s}}$ as a function of $M_{1} / M_{2}$ [7]. Comparing the calculated and published values of $k$ s shows good agreement, i.e., to within $0.1 \%$.

The calculated values of $k_{\mathrm{s}}$ for the Advanced Markus chamber we used to measure the dosimetry of high- $D P P$ electron beams is shown in Fig. 4. This figure shows the two $k_{\mathrm{s}}$ curves at two voltage ratios obtained with the new method (based on Boag's model) and the two corresponding $k_{\mathrm{s}}$ curves calculated using current methods (those of the IAEA and the AAPM). The $k_{\mathrm{s}}$ curves obtained using the new method can be fitted with the following function for practical use:

$$
k_{s}=b_{0}+b_{1}\left(\frac{M_{1}}{M_{2}}\right)+b_{2}\left(\frac{M_{1}}{M_{2}}\right)^{2} .
$$

Here, $b_{0}=1.3890, b_{1}=-0.9705$, and $b_{2}=0.5819$ for polarization voltage ratio $V_{1} / V_{2}=400 / 200$ and $b_{0}=1.0413, b_{1}=-0.1549$, and $b_{2}=0.1157$ for $V_{1} / V_{2}=300 / 100$. In Fig. 4 , the values of $k_{\mathrm{s}}$ obtained with the currently used IAEA TRS-398 and AAPM TG-51 protocols are similar, but they are higher than those obtained with Boag's model. Compared to the $k_{\mathrm{s}}$ value for $M_{1} / M_{2}=1.10$ at $V_{1} / V_{2}=400 / 200$, the results from the IAEA TRS-398 and AAPM TG-51 protocols differed by about $8.9 \%$ and $8.2 \%$, respectively, although no significant difference was found in the range $M_{1} / M_{2}<1.01$.

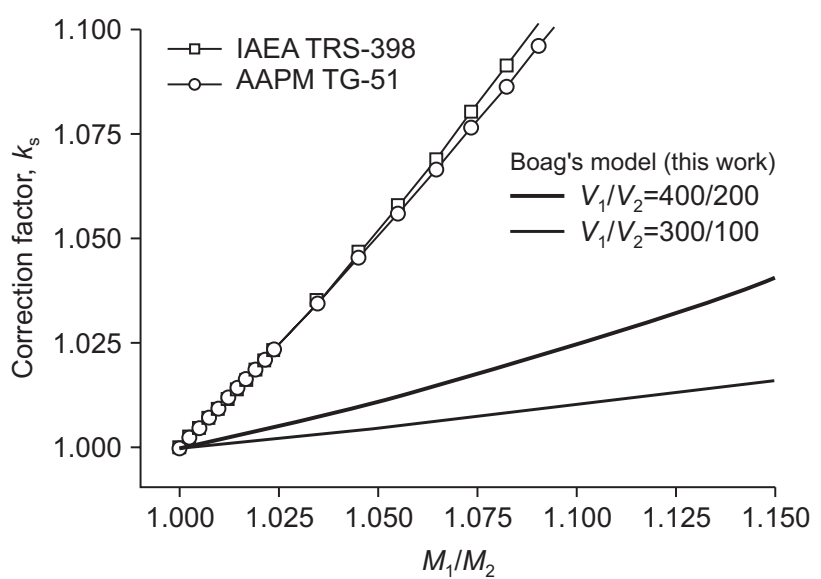

Fig. 4. The ion-recombination correction factors at $V_{1} / V_{2}=400 / 200$ and $V_{1} / V_{2}=300 / 100$ calculated in this work using Boag's model. The correction factors calculated by the conventional methods are included for comparison.

\section{Electron-beam measurements}

The PDD curve measured with radiochromic film in water to determine the beam-quality index $R_{50}\left(=2.4 \mathrm{~g} / \mathrm{cm}^{2}\right)$ is shown in Fig. 5 . The mean electron energy at the phantom surface, $\bar{E}=2.33 R_{50}=5.6 \mathrm{MeV}$, and the most probable energy, $E_{p}=0.22+1.98 R_{p}+0.0025 R_{p}^{2} \approx 6.4 \mathrm{MeV}$, can be determined from the PDD plot in Fig. 5 , where $R_{\mathrm{p}}\left(=3.1 \mathrm{~g} / \mathrm{cm}^{2}\right)$ is defined as the practical range of the electron beam in water [17]. These parameters are similar to those of a clinical electron beam with a nominal energy of $6 \mathrm{MeV}$ [17].

We took into account the 0.2 -cm-thick window made of 


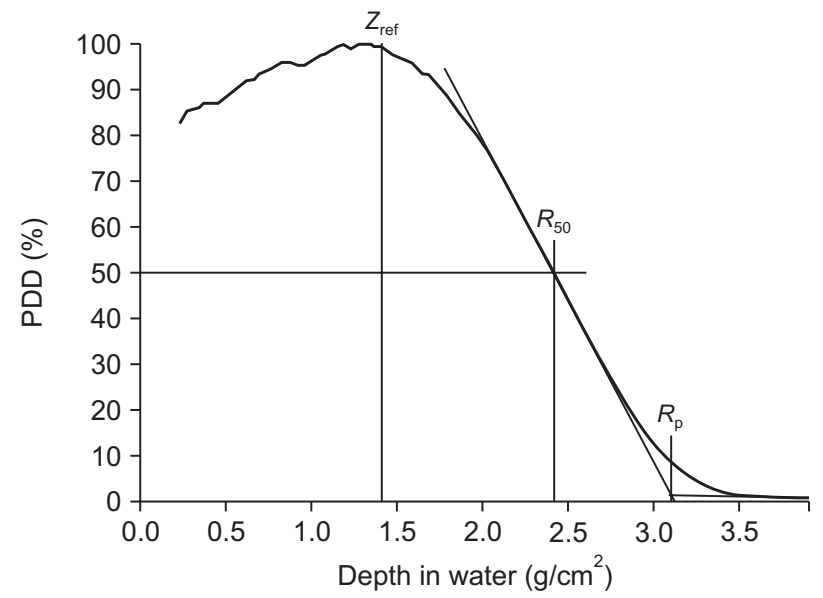

Fig. 5. Measured percentage depth dose (PDD) curve used to determine the beam-quality index $R_{50}$, including the reference depth $\mathrm{z}_{\text {ref }}$ and the practical range $R_{\mathrm{p}}$.

polymethyl methacrylate, with a density of $1.19 \mathrm{~g} / \mathrm{cm}^{3}$, in determining the measurement depth. The reference depth, $z_{\text {ref }}=1.38 \mathrm{~g} / \mathrm{cm}^{2}$, and the beam-quality factor $k_{\mathrm{QQ}_{0}}$ as functions of $R_{50}$ were taken from the tabulated value for the Markus chamber in the TRS-398 protocol. The determined dose in water at the reference depth is listed in Table 1. Because the measured dose $D\left(z_{\text {ref }}\right)$ in the table is for 100 electron-beam pulses at a $50 \mathrm{~Hz}$ repetition rate, the average dose rate is $5.75 \mathrm{~Gy} / \mathrm{s}$, and DPP $\left(z_{\text {ref }}\right)=115 \mathrm{mGy} /$ pulse. The combined standard uncertainty in this measurement is estimated to be $2.4 \%$ (coverage factor $k=1$ ), which includes contributions from the measured value $(0.1 \%)$, the ionrecombination correction factor $(2.0 \%)$, a polarity correction factor $(0.4 \%)$, an air-density correction factor $(0.2 \%)$, an ionization-chamber calibration factor $(0.6 \%)$, a quality factor (1.0\%), and others (0.5\%). Here, we assumed the uncertainty in the ion-recombination correction factor to be the value given by Boag [6].

\section{Discussion}

In applying Boag's model to calculate the ion-recombination correction factor, we verified that the results calculated by our method are the same as those calculated by the iterative numerical method. Our new method can be applied in practical applications without iterative calculations, and it can be presented in the form of the equation currently used in the TRS-398 protocol.
Table 1. Summary of the LINAC operating parameters and electron-beam dosimetry results

\begin{tabular}{lc}
\hline \multicolumn{1}{c}{ Quantity and parameters } & Value \\
\hline LINAC operation & \\
RF average power & $2.5 \mathrm{MW}$ \\
Pulse repetition rate & $50 \mathrm{~Hz}$ \\
Pulse width & $2.5 \mu \mathrm{s}$ \\
Electron-gun heater current & $2.1 \mathrm{~A}$ \\
Number of pulses per irradiation & 100 \\
Dosimetry & \\
Ionization chamber & Advanced Markus \\
Electrometer & PTW UNIDOS \\
Phantom material & Water \\
Beam quality index, $R_{50}$ & $2.4 \mathrm{~g} / \mathrm{cm}^{2}$ \\
Measurement depth, $z_{\text {ref }}$ & $1.38 \mathrm{~g} / \mathrm{cm}^{2}$ \\
$M_{1}(+400 \mathrm{~V})$ & $(9.049 \pm 0.187) \mathrm{nC}$ \\
$M_{2}(+200 \mathrm{~V})$ & $(8.262 \pm 0.217) \mathrm{nC}$ \\
$M_{-}(-400 \mathrm{~V})$ & $(-9.071 \pm 0.078) \mathrm{nC}$ \\
$M_{1} / M_{2}$ & 1.095 \\
$k_{s}\left(M_{1} / M_{2}\right)$ & 1.024 \\
$k_{p o l}$ & 1.001 \\
$k_{T P}$ & 1.029 \\
$M$ corrected & $9.544 \mathrm{nC}$ \\
$N_{D W} Q_{0}$ & $1.308 \mathrm{~Gy} / \mathrm{nC}$ \\
$k_{Q Q_{0}}$ & 0.921 \\
Absorbed dose, $D\left(z_{\text {ref }}\right)$ & $11.50 \mathrm{~Gy}$ \\
Dose-per-pulse, $D P P\left(z_{\text {ref }}\right)$ & $115 \mathrm{mGy} / \mathrm{pulse}$ \\
Combined uncertainty $(k=1)$ & $2.4 \%$ \\
\hline
\end{tabular}

LINAC, linear accelerator.

It is important to note that only the voltage ratio $V_{1} / V_{2}$ is used in the TRS-398 and TG-51 protocols. However, to determine the correction factor, two voltages must be applied separately to use Boag's model. This means, for example, that for the two voltage ratios $V_{1} / V_{2}=400 / 200$ and $V_{1} / V_{2}=300 / 150$, the same correction factor is calculated from the TRS-398 and TG-51 protocols, but the results are different in Boag's model for $M_{1} / M_{2}>1$.3. In addition, the current method is independent of the type of ionization chamber, but Boag's model requires the electrode spacing of the ionization chamber in order to calculate the electric field in the cavity [6]. In high-DPP (>200 mGy/pulse) or FLASH beams, higher values of $M_{1} / M_{2}$ require additional verification. However, performing such additional verifications is currently difficult due to the lack of reported data.

Although several forms of the relationship between high DPP and the ion-collection efficiency of the ioniza- 
tion chambers used for absolute dosimetry were reported $[5,8]$, applying these results directly to obtain dose determinations using an ionization chamber is difficult without performing absolute dosimetry. Film dosimetry may be exploited instead of absolute dosimetry for studying the ion-recombination correction factor for high-DPP beams, although the accuracy is limited. Further, since Boag's theory has not been validated for high-DPP ( $>200 \mathrm{mGy} /$ pulse) beams, in this study we performed the measurements only for a dose rate obtained at $\mathrm{SSD}=100 \mathrm{~cm}$.

\section{Conclusions}

We carried out the present study in order to devise a practical method of applying Boag's improved model for the dosimetry of high-dose-rate electron beams using commercial ionization chambers. This correction method can be applied to DPP ranges up to about $120 \mathrm{mGy} /$ pulse. This upper limit is a suggested value based on the verification of Laitano et al. [7]. It can also be applied to electron beams of higher DPP, subject to independent verifications.

The estimated dose rate of electron beams used in this study is $11.50 \mathrm{~Gy} / \mathrm{s}$, assuming a LINAC pulse repetition rate of $100 \mathrm{~Hz}$. This value is lower than that required in FLASH preclinical studies $[2,3]$. However, the dose rate can be increased significantly by reducing the SSD, as shown in a previous study using our LINAC system [16]. We plan to continue additional studies on the development of correction methods for FLASH beams, eventually applying the results to the construction and commissioning of a FLASH electron-beam irradiation system.

\section{Acknowledgements}

The study was supported by the Dongnam Institute of Radiological and Medical Sciences (DIRAMS) grant funded by the Korea government (MSIT) (No. 50498-2020).

\section{Conflicts of Interest}

The authors have nothing to disclose.

\section{Availability of Data and Materials}

All relevant data are within the paper and its Supporting Information files.

\section{Author Contributions}

Conceptualization: Dong Hyeok Jeong. Data curation: Dong Hyeok Jeong, Kyoung Won Jang. Formal analysis: Heuijin Lim, Sang Koo Kang. Methodology: Dong Hyeok Jeong. Project administration: Dong Hyeok Jeong. Software: Manwoo Lee. Validation: Dong Hyeok Jeong, Kyoung Won Jang. Visualization: Dong Hyeok Jeong, Kyoung Won Jang. Writing-original draft: Dong Hyeok Jeong, Kyoung Won Jang. Writing-review \& editing: Dong Hyeok Jeong, Kyoung Won Jang.

\section{References}

1. Pimpinella M, Andreoli S, De Angelis C, Della Monaca S, D'Arienzo M, Menegotti L. Output factor measurement in high dose-per-pulse IORT electron beams. Phys Med. 2019; 61:94-102.

2. Montay-Gruel P, Petersson K, Jaccard M, Boivin G, Germond JF, Petit B, et al. Irradiation in a flash: unique sparing of memory in mice after whole brain irradiation with dose rates above 100Gy/s. Radiother Oncol. 2017;124:365-369.

3. Schüler E, Trovati S, King G, Lartey F, Rafat M, Villegas $\mathrm{M}$, et al. Experimental platform for ultra-high dose rate FLASH irradiation of small animals using a clinical linear accelerator. Int J Radiat Oncol Biol Phys. 2017;97:195-203.

4. Nisbet A, Thwaites DI. Polarity and ion recombination correction factors for ionization chambers employed in electron beam dosimetry. Phys Med Biol. 1998;43:435-443.

5. McManus M, Romano F, Lee ND, Farabolini W, Gilardi A, Royle G, et al. The challenge of ionisation chamber dosimetry in ultra-short pulsed high dose-rate Very High Energy Electron beams. Sci Rep. 2020;10:9089.

6. Boag JW, Hochhäuser E, Balk OA. The effect of free-electron collection on the recombination correction to ionization measurements of pulsed radiation. Phys Med Biol. 1996;41:885-897.

7. Laitano RF, Guerra AS, Pimpinella M, Caporali C, Petrucci 
A. Charge collection efficiency in ionization chambers exposed to electron beams with high dose per pulse. Phys Med Biol. 2006;51:6419-6436.

8. Petersson K, Jaccard M, Germond JF, Buchillier T, Bochud F, Bourhis J, et al. High dose-per-pulse electron beam dosimetry - a model to correct for the ion recombination in the Advanced Markus ionization chamber. Med Phys. 2017;44: 1157-1167.

9. Pearce JAD. Characterisation of two new ionisation chamber types for use in reference electron dosimetry in the UK. Teddington: National Physical Laboratory. 2004; NPL Report DQL-RD001.

10. Gotz M. Dosimetry of highly pulsed radiation fields. Dresden: Helmholtz-Zentrum Dresden-Rossendorf. 2018; HZDR-090.

11. International Atomic Energy Agency. Absorbed dose determination in external beam radiotherapy: an international code of practice for dosimetry based on standards of absorbed dose to water. Vienna: International Atomic Energy Agency. 2006; IAEA TRS-398.

12. Almond PR, Biggs PJ, Coursey BM, Hanson WF, Huq MS, Nath R, et al. AAPM's TG-51 protocol for clinical reference dosimetry of high-energy photon and electron beams. Med
Phys. 1999;26:1847-1870.

13. Kry SF, Popple R, Molineu A, Followill DS. Ion recombination correction factors (P(ion)) for Varian TrueBeam highdose-rate therapy beams. J Appl Clin Med Phys. 2012;13: 318-325.

14. Lim H, Jo W, Lee DE, Lee M, Kim SH, Shin SW, et al. Status of the DIRAMS C-band standing-wave accelerator for a radiotherapy machine. Paper presented at: 9th Asia Forum for Accelerators and Detectors; 2018 Jan 28-31; Daejeon, Korea. p. 38.

15. Lim H, Lee M, Kim MY, Yi J, Lee M, Kang SK, et al. Measurement of energy parameters for electron gun heater currents and output dose rate for electron beams from a prototype linac. Prog Med Phys. 2016;27:25-30.

16. Jang KW, Lee M, Lim H, Kang SK, Lee SJ, Kim JK, et al. Development of a wide dose-rate range electron beam irradiation system for pre-clinical studies and multi-purpose applications using a research linear accelerator. Prog Med Phys. 2020;31:9-19.

17. Ding GX, Rogers DW. Mean energy, energy-range relationships and depth-scaling factors for clinical electron beams. Med Phys. 1996;23:361-376. 